\title{
A CASE OF ANGIOMATOSIS RETINAE
}

\author{
BY \\ W. NiCCOL \\ GLOUCESTER \\ and \\ R. FOSTER MOORE \\ LONDON
}

THE following case seems to be sufficiently rare to be recorded. The patient was a man, aged 32 years.

.He first consulted Mr. Niccol on May 12, 1933, and was referred to $\mathrm{Mr}$. Foster Moore on June 8.

He was a perfectly healthy individual and, until a few months previously, was acting as an air pilot. He passed the R.A.F. test as pilot in 1931 so that, apart from his own statement, it may safely be concluded that his sight was perfect at that time.

Fourteen days before being seen by $\mathrm{Mr}$. Niccol during an attack of "flu.", he noticed a black spot before the right eye, and for the last five days had been troubled by metamorphopsia.

On examination the left eye was perfect in every way. The condition of the right eye was as follows :-

$R$. V.=6/36. Visual field showed enlargement of the blind spot (Fig. 1) towards the fixation point, but the peripheral field was complete.

Ophthalmoscopic appearances.-(Fig. 2) Immediately adjoining, and partly overlying, the temporal edge of the papilla was a swelling of a peculiar irregular formation, seeming to be composed of a collection of small spherules clustered together, giving somewhat the appearance of a raspberry.

Its general colour was slate-grey; some of the spherules had a reddish or purplish colour, and evidently contained blood.

There were some haemorrhages on the surface, and a few extended along the upper temporal vessels. Rucking of the retina was present, close to the growth and extending towards the macula, and this no doubt accounted for the metamorphopsia. Mr. Niccol estimated that the summit of the growth showed $3 \mathrm{D}$. of swelling. June 12. "The accompanying sketch (see Fig. 2) attempts to show the appearance of the growth on this date. It may enable a conception to be formed of the rather raspberry-like appearance of the swelling, its slaty colour, and the purple colour of some of the superficial prominences. The arterial twig passing over the growth appeared to be partly depleted of blood and formed a light line over the surface of the growth, but did not appear to be definitely implicated in the latter. 


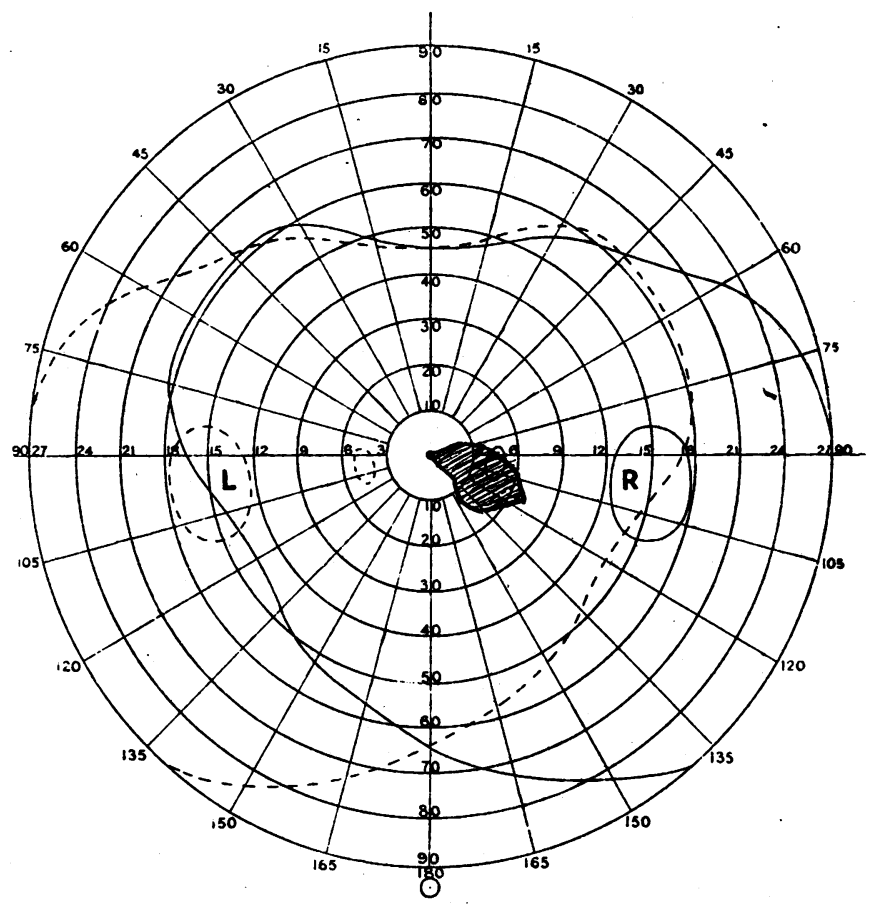

FIG. 1.

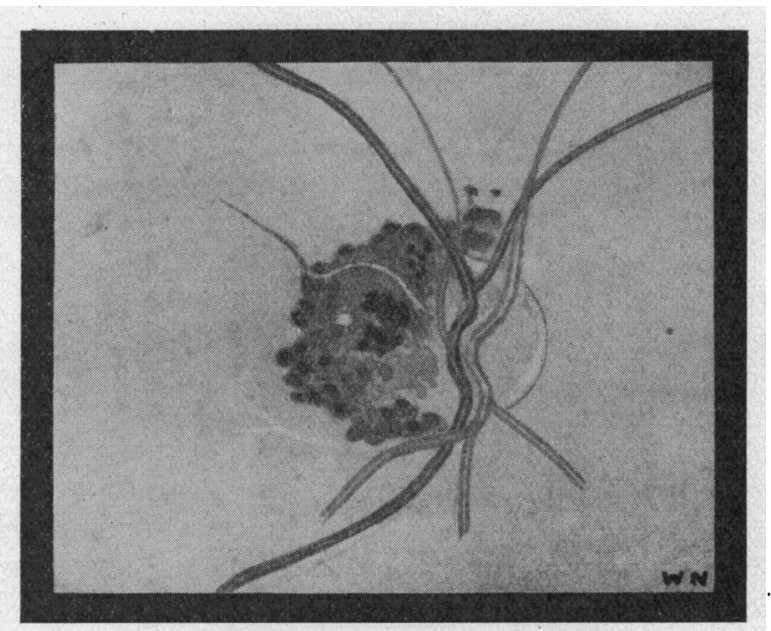

FIG. 2.

E. R., male, aged 32 years.

Right eye enucleated June 26, 1933.

Pathological diagnosis: Angiomatosis retinae. 
"Just below this twig the group of purple bodies had become confluent to some extent, while above it (where retinal haemorrhages had been observed on the earlier date) a number of very small purple spots were present, and two similar spots not previously noted (one apparently separate from the main mass altogether) had appeared above the optic papilla. At the site of previous " rucking" a small, shallow detachment of the retina was noticeable." (W.N.)

There did not appear to be strong reasons for removing the eye at once, and the possibility of dealing with the condition by the insertion of fadon seeds had been suggested to the patient. However, on June 17 , the patient, having consulted a third surgeon, came to the conclusion that the risk of malignancy could not be excluded, and that the eye would become blind, and he urged that it should be removed without further delay. The eye was accordingly removed by $\mathrm{Mr}$. Niccol on June 26.

The following is Mr. H. B. Stallard's report :-

"The neoplasm which invades the temporal half of the optic disc and extends into the adjoining retina on the temporal side is composed of many irregular-shaped spaces of various sizes, lined by endothelial cells and containing blood. At the temporal edge of the optic disc the neoplasm at several points projects forwards through the nerve fibre layer and into the vitreous chamber. The outer nuclear layer of the retina is considerably disorganized by the neoplasm which has compressed and destroyed the rods and cones for about $1 \mathrm{~mm}$. adjacent to the optic disc, and the overlying choroid at this site is flattened. Neuroglial tissue is present between the cavernous spaces, which communicate with branches of the central retinal vessels. At one site there is an area of hyaline degeneration. Some oedema is present in the retinal part of the neoplasm and a thin sheet of blood extends outwards towards the macula between the retinal pigment epithelium and the rods and cones. A few small haemorrhages are present on the summit of the neoplasm over the optic disc."

"The photo-micrographs of transverse sections through the neoplasm and the optic disc illustrate some of its more important histological features. There is no evidence of malignancy or extraocular extension. Pathological diagnosis. Angiomatosis retinae."

The condition is a very rare one and we imagine had been present since birth, but had recently increased in size and involved the macula, and so had given rise to symptoms which led to its discovery.

Without in any way criticising the decision to remove the eye, it is a case which we believe could have been successfully treated by means of radon. 
In order to obtain satisfactory access, division of the external rectus would have been necessary and, from this, disturbance of muscle balance might have ensued ; further, it would not be possible to guarantee immunity from damage by the emanations to the optic nerve and adjoining retina.

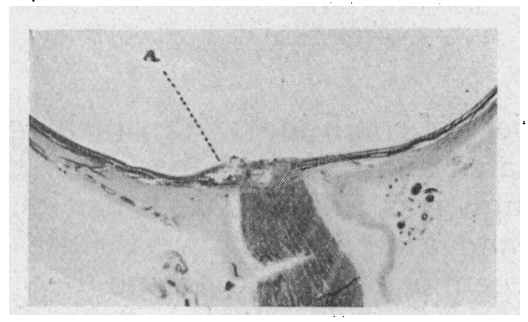

FIG. 3.

Photomicrograph of low power view of transverse section through the globe showing the disposition of the neoplasm (a)..

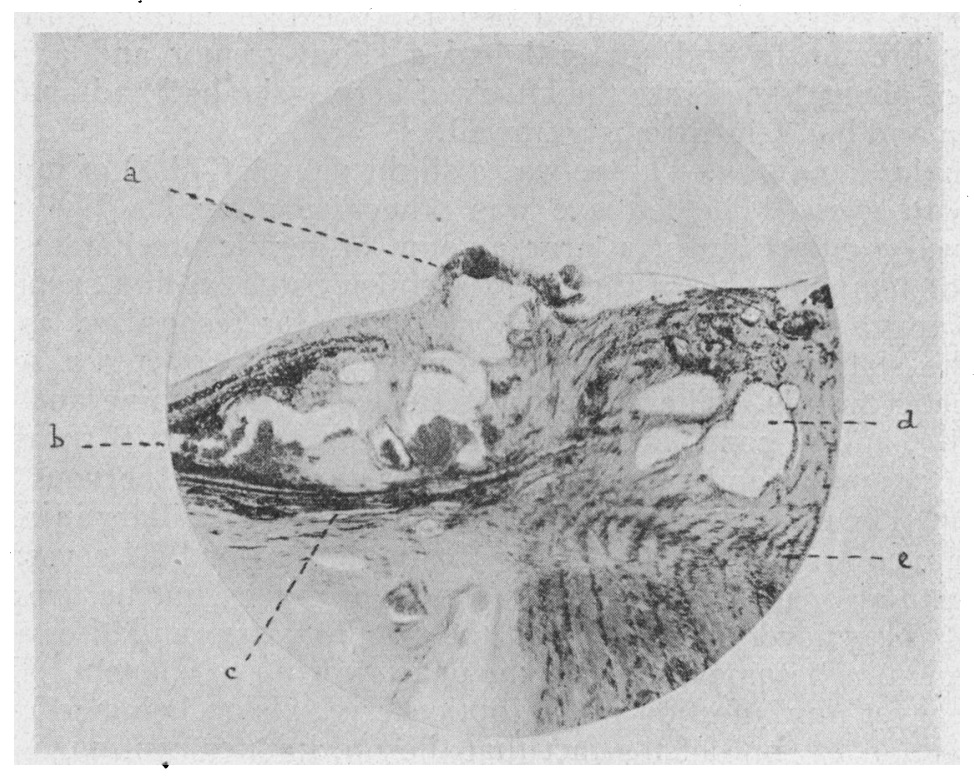

FIG. 4.

Photomicrograph of a transverse section through the temporal half of the optic disc and the adjoining retina and choroid.

a. Part of the neoplasm projecting forwards into the vitteous.

b. The neoplasm invading the outer nuclear layer.

c. Destruction of the rods and cones and the outer nuclear layer at this site.

d. Cavernous spaces in the temporal half of the papilla.

e. Lamina cribrosa. 